TITLE:

\title{
Measurement of spin-precession angles of resonant tunneling neutrons
}

\section{$\operatorname{AUTHOR}(S)$ :}

Hino, M; Achiwa, N; Tasaki, S; Ebisawa, T; Kawai, T; Yamazaki, D

\section{CITATION:}

Hino, M ...[et al]. Measurement of spin-precession angles of resonant tunneling neutrons. PHYSICAL REVIEW A 2000, 61(1): 013607.

ISSUE DATE:

2000-01

URL:

http://hdl.handle.net/2433/39817

RIGHT:

Copyright 2000 American Physical Society 
PHYSICAL REVIEW A, VOLUME 61, 013607

\title{
Measurement of spin-precession angles of resonant tunneling neutrons
}

\author{
Masahiro Hino, ${ }^{1}$ Norio Achiwa, ${ }^{2}$ Seiji Tasaki, ${ }^{1}$ Toru Ebisawa, ${ }^{1}$ Takeshi Kawai, ${ }^{1}$ and Dai Yamazaki ${ }^{3}$ \\ ${ }^{1}$ Research Reactor Institute, Kyoto University, Osaka, 590-0494, Japan \\ ${ }^{2}$ Department of Physics, Kyushu University, Fukuoka, 812-8581, Japan \\ ${ }^{3}$ Department of Nuclear Engineering, Kyoto University, Kyoto, 606-8501, Japan
}

(Received 16 August 1999; published 14 December 1999)

\begin{abstract}
We have succeeded in precisely measuring spin-precession angles of tunneling and nontunneling neutrons through double-rectangular, triple-rectangular, and multirectangular potential barriers represented by a $[\text { Permalloy45(PA)-germanium }(\mathrm{Ge})]^{n}$-PA Fabry-Perot magnetic thin-film resonator for $n=1,2$, and 10 , respectively. The spin-precession angle due to the Fabry-Perot resonator shows the oscillation curve as a function of the incident angles, and the curve is well reproduced by the theoretical phase difference of $\uparrow$ and $\downarrow$ spin neutron wave functions based on the one-dimensional Schrödinger equation. We demonstrate that the amplitude of the curve is proportional to the number of germanium layers (wells) although the transmission probability remains constant.
\end{abstract}

PACS number(s): 03.75.Dg, 03.65.-w, 73.40.Gk

\section{INTRODUCTION}

Recently, we have succeeded in measuring spinprecession angles of neutrons tunneling through a Permalloy45. $\left(\mathrm{Fe}_{55} \mathrm{Ni}_{45}\right)$ ferromagnetic thin film [1-3]. It shows that the spin-precession angle agrees with the stationary-state prediction. That is to say, it is well reproduced by the relative phase difference between $\uparrow$ and $\downarrow$ spin neutron wave functions based on the one-dimensional Schrödinger equation. In this paper we report the experimental results of the spinprecession angle of neutrons through the Fabry-Perot magnetic thin-film resonators. The Fabry-Perot resonator consists of a sequence Permalloy45(PA)-germanium(Ge)-PA with suitable layer thicknesses. In such a Fabry-Perot resonator, $\uparrow$ spin neutrons "feel"' a one-dimensional double-rectangular potential barrier as shown in Fig. 1, and seem to be trapped in quasibound states at resonance condition. On the other hand, $\downarrow$ spin neutrons almost touch one small rectangular potential barrier, and pass through the Fabry-Perot resonator.

An early observation of the quasibound states of neutrons in the double-rectangular potential, represented by the FabryPerot resonator, was performed with an ultracold neutrons [4]. Several authors have investigated neutron optics for resonant tunneling phenomena by means of nonmagnetic Fabry-Perot resonators [5-8]. None has reported the spinprecession angle of resonant tunneling neutrons except for our previous work [9]. Thus, the purpose of this paper is (1) to precisely measure the spin-precession angle of neutron resonant tunneling through double-rectangular, triplerectangular, and multirectangular potential barriers for $\uparrow$ spin neutrons; (2) to compare the measured precession angle with the relative phase difference of $\uparrow$ and $\downarrow$ spin neutron wave functions derived by solving the one-dimensional Schrödinger equation; and (3) to show the relation of the spinprecession angles and transmission probability of $\uparrow$ spin neutrons as a function of incident angles.

\section{BASIC THEORY AND MEASUREMENT METHOD}

\section{A. Spin precession of neutrons} through the Fabry-Perot magnetic resonator

The refraction and reflection of a neutron beam at the surface of a layer are considered as the problem of a rectan- gular potential barrier in a one-dimensional Schrödinger equation [10]. In a magnetic layer, the average nuclear and magnetic potential are given by $V=\left(2 \pi \hbar^{2} / m\right) \rho b_{\text {coh }}$ and $\mu B$, respectively. $m$ and $\mu$ are the neutron mass and the neutron magnetic moment, respectively, and $\rho, b_{\text {coh }}$, and $B$ are the number density of atoms, the average coherent scattering length, and the magnetic induction, respectively. Although the scattering length $b_{\text {coh }}$ is complex in general, the imaginary part is negligible because the magnitude of the imaginary part is $10^{-4}$ or less compared to the coherent part $(V)$ in our experiments.
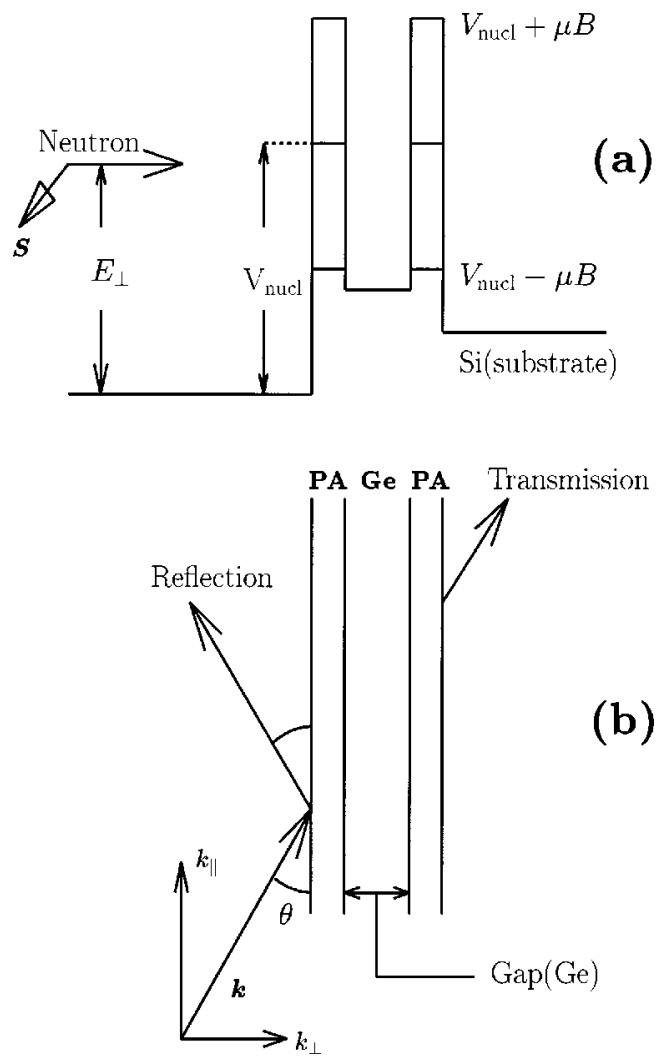

FIG. 1. (a) Potential energy and (b) schematic view for a spinprecessing neutron entering into a Fabry-Perot magnetic thin-film resonator at an incident angle $\theta$. 
Let us consider the spin precession of neutrons transmitted through a Fabry-Perot magnetic thin-film resonator as shown in Fig. 1. The state of the spin-precessing neutron is represented as a coherent superposition of eigenstates of $\uparrow$ and $\downarrow$ spin neutrons $[11,12]$. In the transmission process, the Hamiltonian is diagonal and the direction of the quantization axis does not change. The stationary wave function in the $\alpha$ th region (layer) can be described as

$$
|\psi\rangle=\left(\begin{array}{c}
\psi_{+}(y) \\
\psi_{-}(y)
\end{array}\right)=\left(\begin{array}{c}
A_{+} e^{i q_{\alpha(+)} y}+B_{+} e^{-i q_{\alpha(+)} y} \\
A_{-} e^{i q_{\alpha(-1)} y}+B_{-} e^{-i q_{\alpha(-)} y}
\end{array}\right),
$$

where $q_{\alpha( \pm)}=\sqrt{2 m\left(E_{\perp}-V_{\alpha} \bar{\mp} \mu B_{\alpha}\right)} / \hbar$ and $E_{\perp}=\hbar^{2} k_{\perp}^{2} / 2 m$. $k_{\perp}$ and $q_{\alpha}$ are normal components of the wave vector in vacuum and the $\alpha$ th region, respectively, and $V_{\alpha}$ and $B_{\alpha}$ are the average nuclear and magnetic potentials in the $\alpha$ th region, respectively. The subscripts + and - indicate the neutron of $\uparrow$ and $\downarrow$ spin, respectively. Here region 1 is vacuums (air), region $n$ is substrate, and regions 2 to $n-1$ are layers in the Fabry-Perot resonator. From the boundary conditions where $\psi$ and $d \psi / d y$ are continuous, the transfer matrix is described as [13]

$$
\left(\begin{array}{l}
1 \\
r
\end{array}\right)=\left(\begin{array}{ll}
M_{11} & M_{12} \\
M_{21} & M_{22}
\end{array}\right)\left(\begin{array}{c}
t \\
0
\end{array}\right) \text {. }
$$

The transmission and specular reflection coefficients $t$ and $r$ are given by $t=1 / M_{11}$ and $r=M_{21} / M_{11}$. The transfer matrix is given by [14]

$$
\begin{aligned}
& \hat{M}=\hat{D}^{-1}\left(q_{1}\right)\left(\prod_{j=2}^{N-1} \hat{D}\left(q_{j}\right) \hat{P}\left(q_{j}, d_{j}\right) \hat{D}^{-1}\left(q_{j}\right)\right) \hat{D}\left(q_{N}\right), \\
& \hat{D}\left(q_{j}\right)=\left(\begin{array}{cc}
1 & 1 \\
q_{j} & -q_{j}
\end{array}\right), \quad \hat{P}\left(q_{j}, d_{j}\right)=\left(\begin{array}{cc}
e^{-i q_{j} d_{j}} & 0 \\
0 & e^{i q_{j} d_{j}}
\end{array}\right),
\end{aligned}
$$

where $\hat{D}\left(q_{\alpha}\right)$ are the transmission matrices and $\hat{P}\left(q_{\alpha}, d_{\alpha}\right)$ are the propagation matrices for the $\alpha$ th region, and $d_{\alpha}$ is the width of the $\alpha$ th region (layer).

The transmitted stationary wave function is described as

$$
\begin{gathered}
\left|\psi_{\mathrm{tr}}\right\rangle=\left(\begin{array}{l}
t_{+} e^{i k y} \\
t_{-} e^{i k y}
\end{array}\right) \\
t_{ \pm}=T_{ \pm}^{1 / 2} e^{i \Delta \phi_{ \pm}} e^{-i k d} e^{\mp i \delta / 2},
\end{gathered}
$$

where $\Delta \phi$ and $T$ are the additional phase and the transmission probabilities through the resonator, respectively, $d$ is the total thickness of the resonator, and $\delta$ is the incident spin precession angle at the surface of the resonator.

The normalized expectation values of a neutron transmitted through the resonator $\left\langle S_{x} ; \operatorname{tr}\right\rangle,\left\langle S_{y} ; \operatorname{tr}\right\rangle$, and $\left\langle S_{z} ; \operatorname{tr}\right\rangle$ are given by

$$
\left\langle S_{x} ; \mathrm{tr}\right\rangle=\frac{\hbar}{2}\left\langle\psi_{\mathrm{tr}}\left|\sigma_{x}\right| \psi_{\mathrm{tr}}\right\rangle=\hbar \cos \left(\Delta \phi_{+}-\Delta \phi_{-}-\delta\right) \frac{\sqrt{T_{+} T_{-}}}{T_{+}+T_{-}},
$$

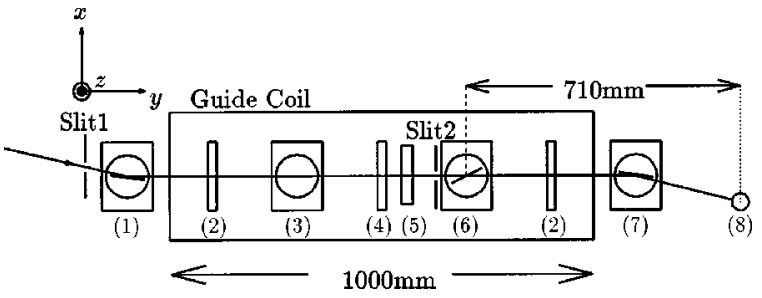

FIG. 2. Schematic layout of the neutron spin interferometer at JRR-3M. (1) Polarizer, (2) $\pi / 2$ spin flipper coil, (3) precession coil I (PC1), (4) $\pi$ spin flipper coil, (5) accelerator coil, (6) precession coil II (PC2) and a Fabry-Perot magnetic resonator (sample), (7) analyzer, (8) ${ }^{3} \mathrm{He}$ detector.

$$
\begin{aligned}
&\left\langle S_{y} ; \operatorname{tr}\right\rangle=\frac{\hbar}{2}\left\langle\psi_{\mathrm{tr}}\left|\sigma_{y}\right| \psi_{\mathrm{tr}}\right\rangle \\
&=-\hbar \sin \left(\Delta \phi_{+}-\Delta \phi_{-}-\delta\right) \frac{\sqrt{T_{+} T_{-}}}{T_{+}+T_{-}} \\
&\left\langle S_{z} ; \operatorname{tr}\right\rangle=\frac{\hbar}{2}\left\langle\psi_{\mathrm{tr}}\left|\sigma_{z}\right| \psi_{\mathrm{tr}}\right\rangle=\frac{\hbar}{2} \frac{T_{+}-T_{-}}{T_{+}+T_{-}}
\end{aligned}
$$

where $\sigma_{x}, \sigma_{y}$, and $\sigma_{z}$ are the Pauli spin matrices. This relative phase difference $\Delta \phi_{+}-\Delta \phi_{-}$is equivalent to the additional spin-precession angle $\Omega$ of the transmitted neutron through the resonator. Solving Eq. (2.3), we can find the coefficient $t$ and predict the spin-precession angle due to the Fabry-Perot magnetic resonator.

\section{B. Measurement of the additional spin-precession angle by means of the NSE method}

The neutron spin echo (NSE) method was proposed by F. Mezei [15]. The essential feature of the NSE method has been well explained with the Larmor precession represented as a classical image of the spin precession of neutrons [16]. The amplitude of the NSE signal is given as a function of the $\delta N$ which is the difference between the numbers of the Larmor precession before and after a $\pi$ flipper coil. In our configuration, shown in Fig. 2, $\delta N$ is described by

$$
\begin{aligned}
\delta N & =N_{0}-N_{1}-N_{2}-\Delta N \\
& =\frac{\gamma L}{2 \pi}\left(\frac{H_{0} l_{0}}{\mathrm{v}_{0}}-\frac{H_{1} l_{1}}{\mathrm{v}_{1}}-\frac{H_{2} l_{2}}{\mathrm{v}_{1}}\right)-\Omega / 2 \pi,
\end{aligned}
$$

where $\gamma_{L}=2 \mu / \hbar=29.16 \mathrm{kHz} / \mathrm{mT}, \Delta N$ is the additional spin precession due to a sample, $N$ is the number of the Larmor precession, $l$ is the length of the magnetic field $H$, and $\mathrm{v}$ is the neutron velocity. The numbers 0,1 , and 2 indicate the situations in the precession coil I (PC1), the precession coil II (PC2), and the accelerator coil, respectively.

Figure 3 shows a typical NSE signal without a sample. In this experiment, $H_{0}, H_{1}, l_{0}, l_{1}$, and $l_{2}$ are constant. The NSE signal is, hence, measured as a function of the current $\mathrm{H}_{2}$ of the accelerator coil. One period of the signal corresponds to one turn of the Larmor precession. In transmission experiments, $\mathrm{v}_{0}=\mathrm{v}_{1}$; therefore, a shift of the NSE signal with and without the sample at an incident angle is equiva- 


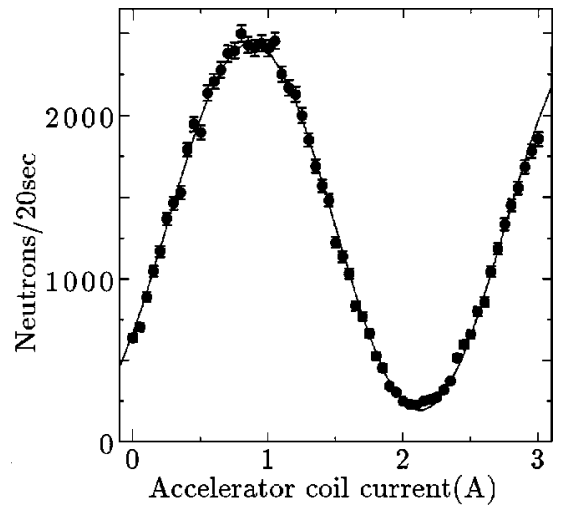

FIG. 3. Typical NSE signal measured without a sample as a function of the accelerator coil current.

lent to an average additional angle of the spin precession of neutrons through the sample at the incident angle. Thus, the additional spin-precession angles $\Omega$ due to the sample are derived from the shifts of NSE signals as a function of the incident angles. Using the magnetic Fabry-Perot resonator as the sample, we measured precisely the spin-precession angle of neutron resonant tunneling through double-rectangular, triple-rectangular, and multirectangular potential barriers for $\uparrow$ spin neutrons.

\section{EXPERIMENTAL RESULTS AND DISCUSSIONS}

\section{A. Experimental procedures}

We have prepared four kinds of Fabry-Perot thin-film resonators, as shown in Table I. The material of the magnetic layers is Permalloy45( $\left(\mathrm{Fe}_{55} \mathrm{Ni}_{45}\right)$ which is magnetically soft. The Fabry-Perot resonator was evaporated on a polished silicon wafer in an applied magnetic field of $14 \mathrm{mT}$ in order to saturate the magnetic layers under a lower magnetic field [17]. Each layer thickness was measured by a quartz crystal oscillator during evaporation [18]. The silicon wafers were disks with a 75-mm diameter and a 3-mm thickness.

In the $[\mathrm{PA}-\mathrm{Ge}]^{n}$-PA Fabry-Perot resonator, the $\uparrow$ spin neutron "feels" double-rectangular $(n=1)$, triplerectangular $(n=2)$, or multirectangular potential $(n=10)$ barriers, although the $\downarrow$ spin neutron almost touches one small rectangular potential barrier. The experiments were carried out with the cold neutron spin interferometer (NSIJAERI) [19] installed at the C3-1-2 beam port of the JRR-3M reactor at the Japan Atomic Energy Research Institute (JAERI). The incident wavelength resolution and the divergent angle were 1.26 \pm 0.044 [full width at half maximum (FWHM)] nm and $1.0 \mathrm{mrad}$, respectively. The strength of the magnetic field at the sample position (PC2) for the NSI-JAERI was $2 \mathrm{mT}$. For the simulation of the relative phase difference of $\uparrow$ and $\downarrow$ spin, it is necessary to evaluate the correct values of the average nuclear and magnetic potential in the magnetic and nonmagnetic layers. These potential values can be estimated by the best fitting of the measured transmission probabilities of the $\uparrow$ and $\downarrow$ spin neutron through the Fabry-Perot resonator with simulation. The transmission experiments were carried out with configurations of the NSI-JAERI without the analyzer. At the incident angles below $2.3^{\circ}(3 / 75 \mathrm{rad})$, all neutrons through the substrate come from the edge of the substrate because the maximum length of the silicon substrate is $75 \mathrm{~mm}$ and the thickness is $3.0 \mathrm{~mm}$.

The direction of the neutrons from the edge of the substrate slanted $\theta_{\Delta}$ to the incident neutron beam direction, where $\theta_{\Delta}$ is the difference between the incident angle and the refractive angle in the substrate. $\theta_{\Delta}$ increases when the incident angle is smaller. Therefore we moved the detector along the $x$ direction shown in Fig. 2 when the incident angle is smaller than $\left(0.9^{\circ}\right)$.

\section{B. Double-rectangular potential case}

Figure 4(a) shows the transmission probabilities of $\uparrow$ and $\downarrow$ spin neutrons through only silicon substrate. Figures 4(b) and 4(c) show those through the PA(20 nm)-Ge(40 nm)$\mathrm{PA}(20 \mathrm{~nm})$ Fabry-Perot resonator, respectively. In this Fabry-Perot resonator, $\uparrow$ spin neutrons "feel" a onedimensional double-rectangular potential barrier. The closed and open circles indicate experimental transmission probabilities of $\uparrow$ and $\downarrow$ spin neutrons, respectively, as a function of the incident angle $\theta$. As shown in Fig. 4(a), transmission probabilities of $\uparrow$ and $\downarrow$ spin neutrons through the silicon substrate agree with each other. We confirm that the substrate does not affect the spin-precession angle, and that these transmission probabilities remain constant at the incident angles which are larger than $0.8^{\circ}$. In Figs. 4(b) and 4(c), the lines indicate the theoretical values calculated from Eq. (2.4) for $\uparrow$ and $\downarrow$ spin neutrons, respectively, including the incident wavelength distribution at the NSI-JAERI. These experimental data are well reproduced by the theoretical lines, where the values of the average nuclear and magnetic potentials evaluated in Fig. 4 are shown in Table I.

Figure 5(a) shows the spin-precession angle of neutrons transmitted through the PA(20 nm)-Ge(40 nm)-PA(20 nm) Fabry-Perot resonator. The closed circles indicate measured

TABLE I. Measured parameters of the Fabry-Perot magnetic resonator in transmission experiments. $V_{\mathrm{PA}}$, $V_{\mathrm{Ge}}$, and $V_{\mathrm{Si}}$ indicate values of the nuclear potential of Permalloy45 $\left(\mathrm{Fe}_{55} \mathrm{Ni}_{45}\right)$, germanium, and silicon, respectively.

\begin{tabular}{lccccc}
\hline \hline Fabry-Perot resonator & $V_{\mathrm{PA}}(\mathrm{neV})$ & $|\mu B|(\mathrm{neV})$ & $B(\mathrm{~T})$ & $V_{\mathrm{Ge}}(\mathrm{neV})$ & $V_{\mathrm{Si}}(\mathrm{neV})$ \\
\hline $\mathrm{PA}(20 \mathrm{~nm})-\mathrm{Ge}(40 \mathrm{~nm})-\mathrm{PA}(20 \mathrm{~nm})$ & 224 & 87.4 & 1.45 & 94.0 & 54.0 \\
$\mathrm{PA}(15 \mathrm{~nm})-\mathrm{Ge}(80 \mathrm{~nm})-\mathrm{PA}(15 \mathrm{~nm})$ & 215 & 99.5 & 1.65 & 94.0 & 54.0 \\
{$[\mathrm{PA}(10 \mathrm{~nm})-\mathrm{Ge}(80 \mathrm{~nm})]^{2}-\mathrm{PA}(10 \mathrm{~nm})$} & 217 & 96.5 & 1.60 & 94.0 & 54.0 \\
{$[\mathrm{PA}(20 \mathrm{~nm})-\mathrm{Ge}(40 \mathrm{~nm})]^{10}-\mathrm{PA}(20 \mathrm{~nm})$} & 224 & 84.4 & 1.40 & 94.0 & 54.0 \\
\hline \hline
\end{tabular}



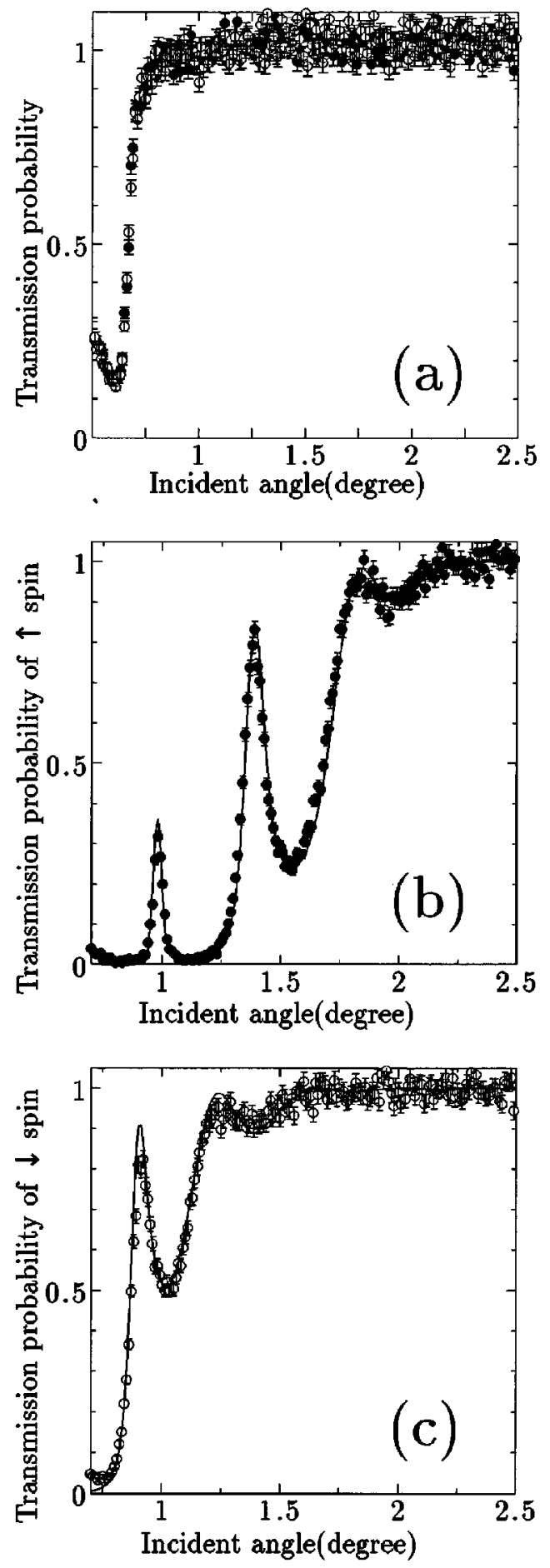

FIG. 4. Transmission probabilities of $\uparrow$ and $\downarrow$ spin neutrons through (a) only silicon substrate as a function of the incident angle. The transmission probabilities of $\uparrow$ and $\downarrow$ spin neutrons through (b) $\uparrow$ and (c) $\downarrow$ spin neutrons through the PA $(20 \mathrm{~nm})-\mathrm{Ge}(40 \mathrm{~nm})$ $\mathrm{PA}(20 \mathrm{~nm})$ Fabry-Perot resonator as a function of the incident angle.

shifts of NSE signals and were obtained by a least-squares fitting of a cosine function to the NSE signals. The dotted vertical line indicates the critical angle of $\uparrow$ spin neutrons for Permalloy45. In both the tunneling and nontunneling regions for $\uparrow$ spin neutrons, the measured spin-precession angle os-
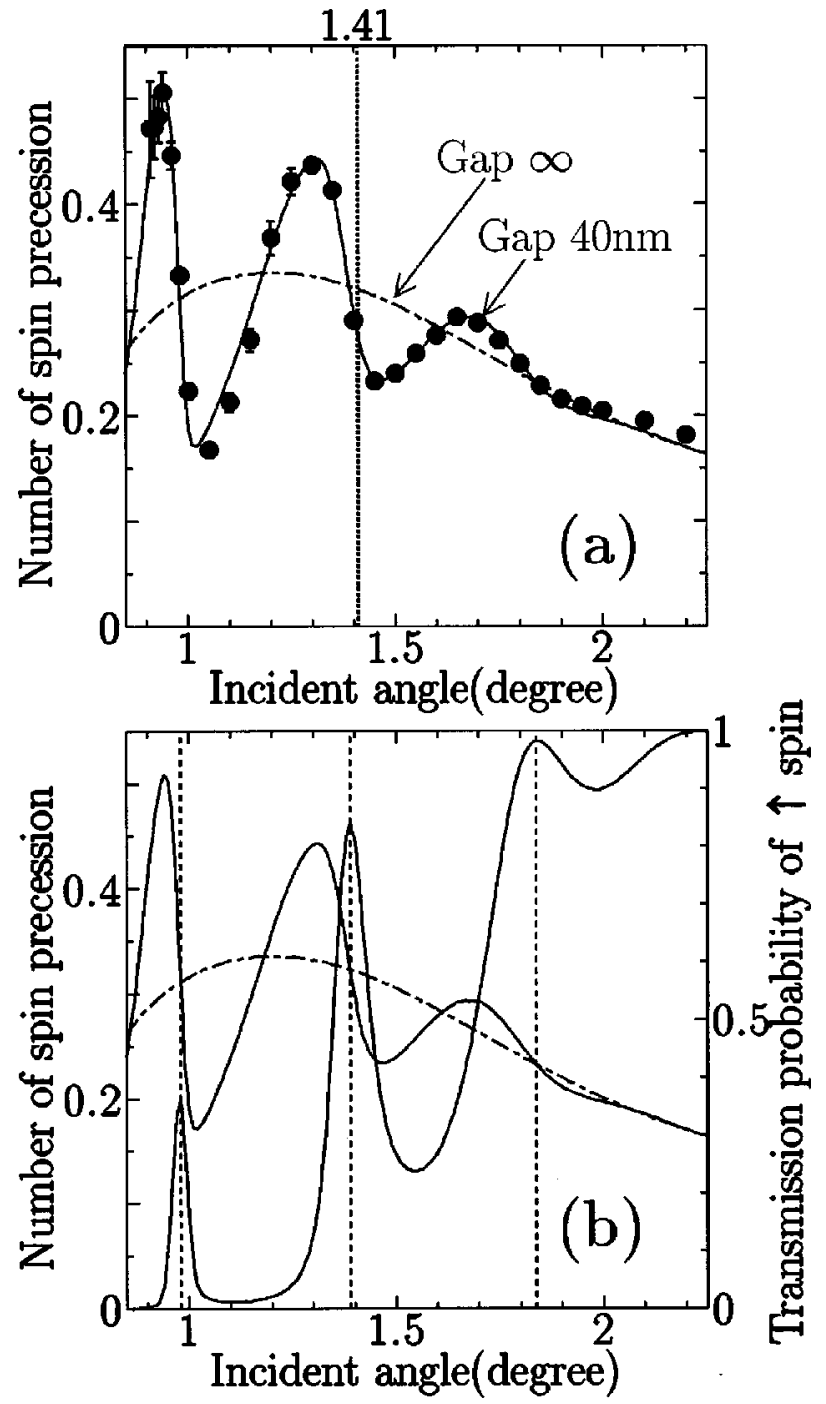

FIG. 5. Spin precession of neutrons transmitted through the PA $(20 \mathrm{~nm})-\mathrm{Ge}(X \mathrm{~nm})-\mathrm{PA}(20 \mathrm{~nm})$ Fabry-Perot resonator for $X$ $=40$ and $\infty$, respectively, as a function of the incident angle.

cillates as a function of the incident angle and is well reproduced by the theoretical relative phase difference of $\uparrow$ and $\downarrow$ spin neutron wave functions calculated from Eq. (2.4). The broken line indicates simulated spin-precession angles through $\mathrm{PA}(20 \mathrm{~nm})-\mathrm{Ge}(\infty)-\mathrm{PA}(20 \mathrm{~nm})$ as a nonresonance case. The spin-precession angle for the PA(20 nm)-Ge( $\infty)$ PA(20 nm) calculated by adding two spin-precession angles that are the relative phase difference of $\uparrow$ and $\downarrow$ spin are derived by solving a one-dimensional Schrödinger equation for $\operatorname{PA}(20 \mathrm{~nm})-\mathrm{Ge}(2 \mu \mathrm{m})$ and $\mathrm{Ge}(2 \mu \mathrm{m})-\mathrm{PA}(20 \mathrm{~nm})-\mathrm{Si}(2$ $\mu \mathrm{m})$ magnetic layers, respectively. Since the spin-precession angle does not depend on the thicknesses of the nonmagnetic layers for these layer systems that have only one magnetic layer, the value of the thickness $(2 \mu \mathrm{m})$ can be considered as infinity. The simulated spin-precession angle for the gap $\infty$ shows the average of the oscillation curve for the gap $40 \mathrm{~nm}$. In this incident wavelength distribution $(\delta \lambda / \lambda=3.5 \%)$, the simulated spin-precession angle for the gap $\geqslant 1 \mu \mathrm{m}$ agreed with that for the gap $\infty$. We unify Figs. 4(b) and 5(a) to Fig. 5 (b) in order to show the relation of the transmission prob- 

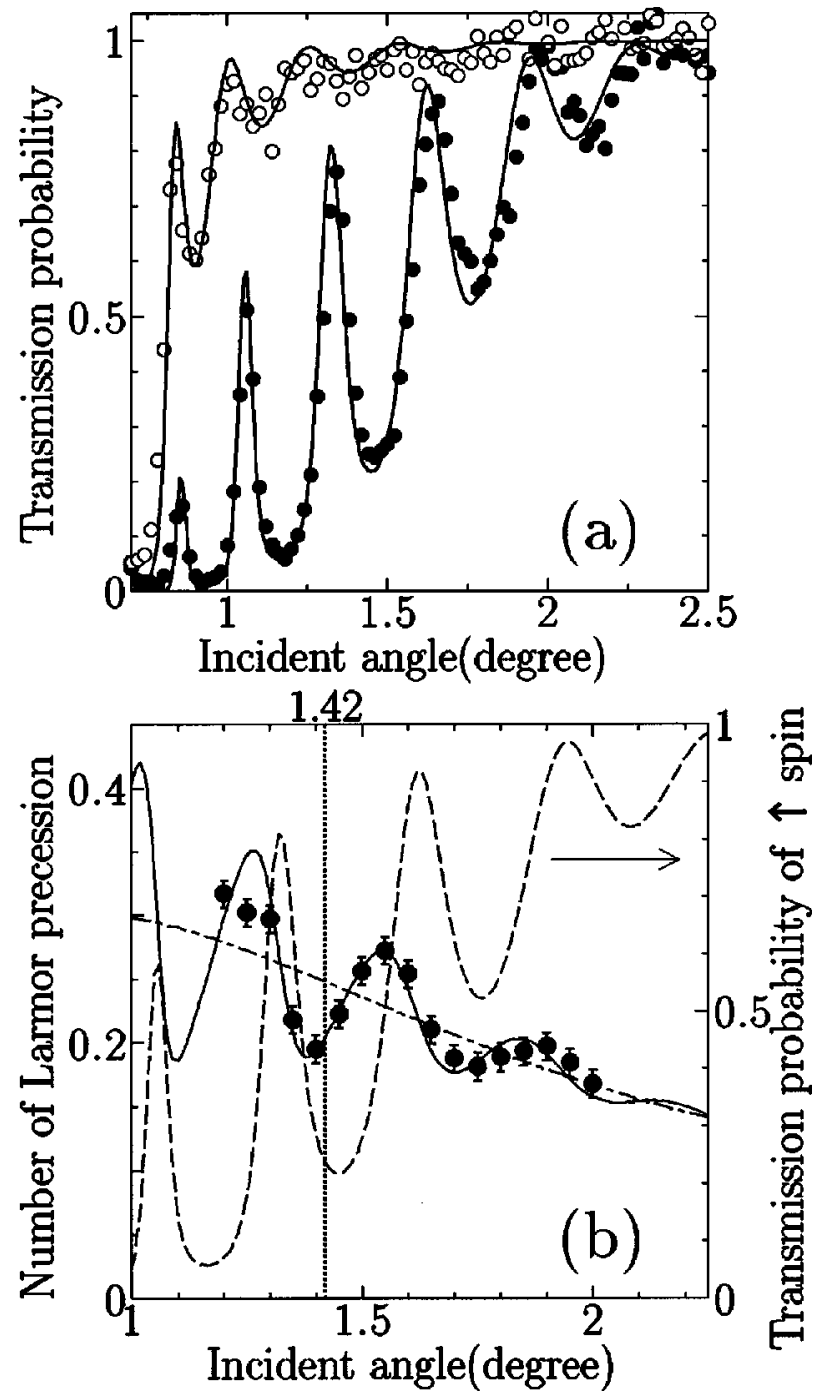

FIG. 6. (a) Transmission probabilities of $\uparrow$ and $\downarrow$ spin neutrons through the PA(15 nm)-Ge(80 nm)-PA(15 nm) Fabry-Perot resonator as a function of the incident angle. (b) The spin precession of neutrons due to the Fabry-Perot resonator as a function of the incident angle.

ability and the number of the spin precession. At the incident angles for peaks of the transmission probability, the spinprecession angles of resonant and nonresonant tunneling neutrons are the same values. From the phase point of view, we can interpret that the resonant tunneling phenomenon appears when no reflected wave seems to be in the well although the probability density of the $\uparrow$ spin neutron reflected by the second wall is not zero in the well.

Figure 6(a) shows the transmission probabilities of $\uparrow$ and $\downarrow$ spin neutrons through the $\mathrm{PA}(15 \mathrm{~nm})-\mathrm{Ge}(80 \mathrm{~nm})-\mathrm{PA}(15$ nm) Fabry-Perot resonator. These closed and open circles indicate the experimental transmission probabilities of $\uparrow$ and $\downarrow$ spin neutrons, respectively, and are also well reproduced by the theoretical lines calculated from Eq. (2.4), including the effects of silicon substrate and the incident wavelength distribution. It shows that the period of the peaks shown in Fig. 6(a) becomes shorter than that shown in Fig. 4(b) and the period is almost proportional to the inverse of the well
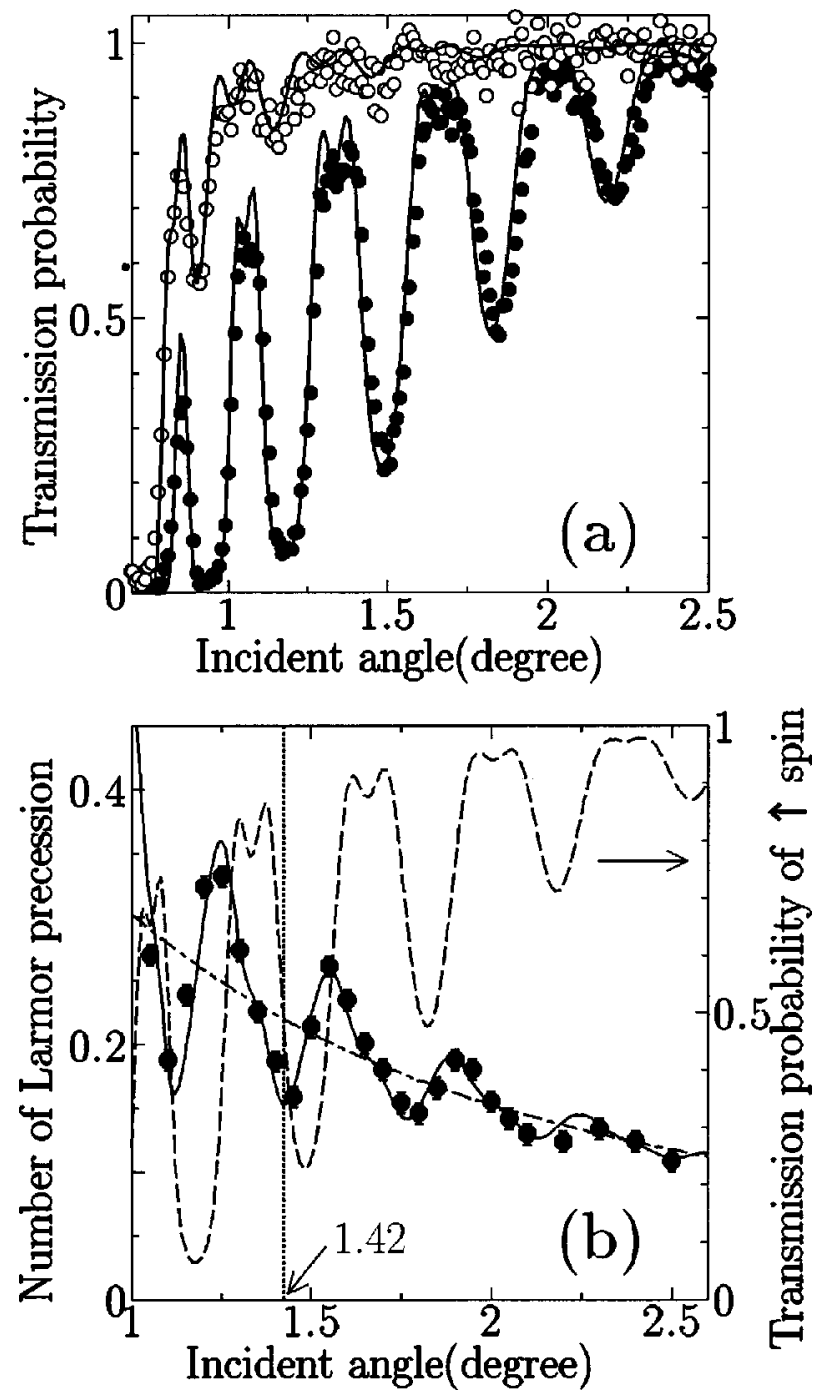

FIG. 7. (a) Transmission probabilities of $\uparrow$ and $\downarrow$ spin neutrons through the $[\mathrm{PA}(10 \mathrm{~nm})-\mathrm{Ge}(80 \mathrm{~nm})]^{2}-\mathrm{PA}(10 \mathrm{~nm})$ Fabry-Perot resonator as a function of the incident angle. (b) The spin precession of neutrons due to the Fabry-Perot resonator as a function of the incident angle.

thickness. The average nuclear and magnetic potentials evaluated in Fig. 6 are also shown in Table I. In Table I, a slight deviation of the potential values was observed. The nominal average nuclear and magnetic potentials for iron are 209 and $131 \mathrm{neV}$, respectively, and those potentials for nickel are 245 and $38.5 \mathrm{neV}$, respectively [20]. The deviation might be the result of the deviation of concentration of iron and nickel in the films. Figure 6(b) shows the spinprecession angle of neutrons transmitted through the FabryPerot resonator. The measured closed circles are also well reproduced by the theoretical relative phase difference of $\uparrow$ and $\downarrow$ spin neutron wave functions calculated from Eq. (2.4). These broken lines indicate simulated spin-precession angles through PA(15 nm)-Ge( $\infty)-\mathrm{PA}(15 \mathrm{~nm})$ as the nonresonance case. The precession angle for $\operatorname{PA}(15 \mathrm{~nm})-\mathrm{Ge}(\infty)-\mathrm{PA}(15 \mathrm{~nm})$ was calculated in the same way as Fig. 4. The critical angle of Permalloy 45 for $\uparrow$ spin neutron is $1.42^{\circ}$. As well as Fig. 5, the transmission probability of the $\uparrow$ spin becomes maximum 

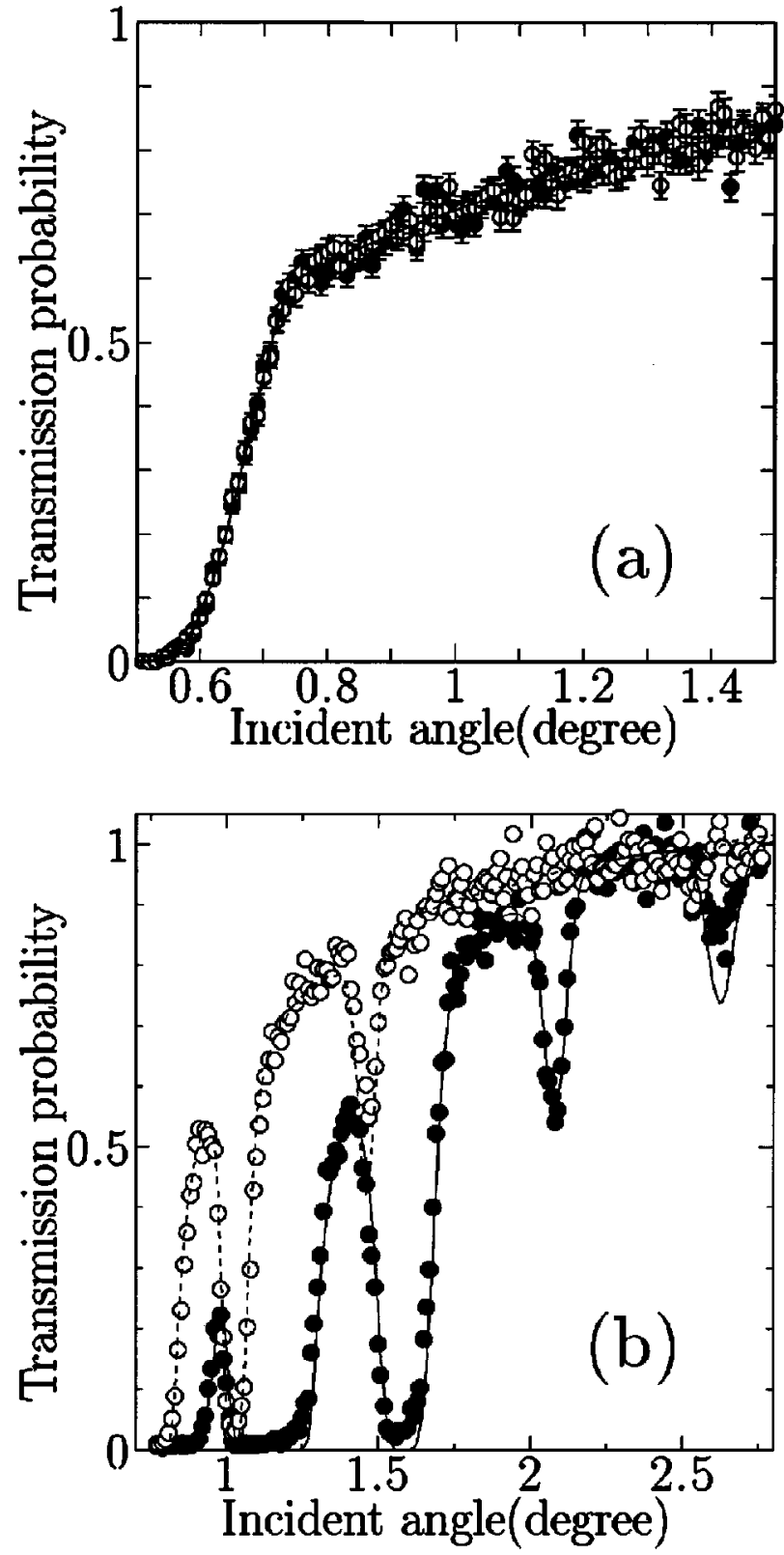

FIG. 8. Transmission probabilities of $\uparrow$ and $\downarrow$ spin neutrons through (a) only silicon substrate and (b) a $[\mathrm{PA}(20 \mathrm{~nm})-\mathrm{Ge}(40 \mathrm{~nm})]^{10}-\mathrm{PA}(20 \mathrm{~nm})$ Fabry-Perot resonator as a function of the incident angle.

at the incident angles where the spin-precession angle of resonant and nonresonant tunneling neutrons agreed.

\section{Triple- and multirectangular potential cases}

Figure 7(a) shows the transmission probabilities of $\uparrow$ and $\downarrow$ spin neutrons through the [PA $(10 \mathrm{~nm})$ $-\mathrm{Ge}(80 \mathrm{~nm})]^{2}-\mathrm{PA}(10 \mathrm{~nm})$ Fabry-Perot resonator. In this Fabry-Perot resonator $\uparrow$ spin neutrons touch a onedimensional triple-rectangular potential barrier. The closed and open circles indicate the experimental transmission probabilities of $\uparrow$ and $\downarrow$ spin neutrons, respectively, and are also well reproduced by the theoretical lines calculated from Eq. (2.4), including the effects of silicon substrate and the inci-

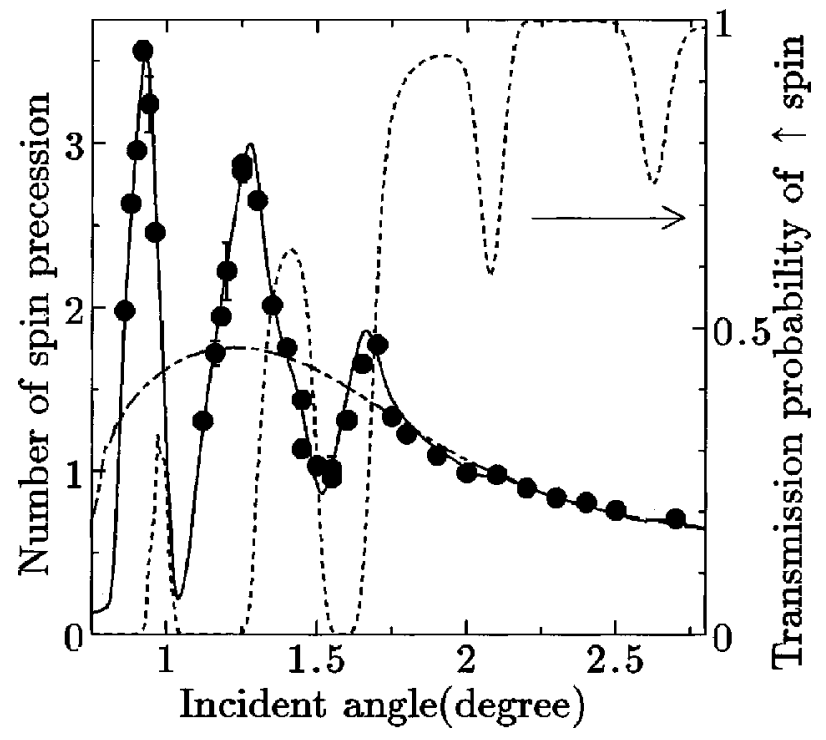

FIG. 9. Spin precession of neutrons transmitted through the $[\mathrm{PA}(20 \mathrm{~nm})-\mathrm{Ge}(40 \mathrm{~nm})]^{10}$-PA $(20 \mathrm{~nm})$ Fabry-Perot resonator as a function of the incident angle.

dent wavelength distribution. The values of the average nuclear and magnetic potentials evaluated in Fig. 7(a) are shown in Table I. The transmission probability of the $\uparrow$ spin neutron for the double-rectangular potential case as shown in Fig. 4(b) is split into two for the triple-rectangular potential case at resonance conditions. It is considered as two energy levels, whose difference is very small, that exist in a quasibound state for the triple-rectangular potential barrier. These results can also be explained by the Airy formula [21] and the detailed explanation for unpolarized neutrons was discussed by Steyerl and co-workers [5].

Figure 7(b) shows the spin-precession angle of neutrons through the $[\mathrm{PA}(10 \mathrm{~nm})-\mathrm{Ge}(80 \mathrm{~nm})]^{2}-\mathrm{PA}(10 \mathrm{~nm})$ FabryPerot resonator. The measured closed circles are also well reproduced by the theoretical relative phase difference of $\uparrow$ and $\downarrow$ spin neutron wave functions calculated from Eq. (2.4). These broken lines indicate simulated spin-precession angles through $[\mathrm{PA}(10 \mathrm{~nm})-\mathrm{Ge}(\infty)]^{2}-\mathrm{PA}(10 \mathrm{~nm})$ as nonresonance cases. The precession angle for [PA(10 nm)$\mathrm{Ge}(\infty)]^{2}-\mathrm{PA}(10 \mathrm{~nm})$ was calculated by adding twice the spinprecession angles of the relative phase difference due to $\mathrm{PA}(15 \mathrm{~nm})-\mathrm{Ge}(2 \mu \mathrm{m})$ and $\mathrm{Ge}(2 \mu \mathrm{m})-\mathrm{PA}(15 \mathrm{~nm})-\mathrm{Si}(2 \mu \mathrm{m})$. Each cross point corresponds to the small grooves of peaks for the transmission probability of the $\uparrow$ spin.

Figure 8(a) shows the transmission probabilities of $\uparrow$ and $\downarrow$ spin neutrons through only silicon substrate, and Fig. 8(b) shows those through the [PA $(20 \mathrm{~nm})$ $\mathrm{Ge}(40 \mathrm{~nm})]^{10}-\mathrm{PA}(20 \mathrm{~nm})$ Fabry-Perot resonator, respectively. Here we call the multirectangular potential barrier as the one-dimensional 11-ply potential barrier from this FabryPerot resonator. In this experiment, we did not move the detector along the $x$ direction shown in Fig. 2, and the neutron transmission intensities were observed at a fixed detector position. Therefore, the intensities of neutrons as shown in Fig. 8(a) are reduced at the incident angles below $0.75^{\circ}$, which is higher than the critical angle of silicon substrate 


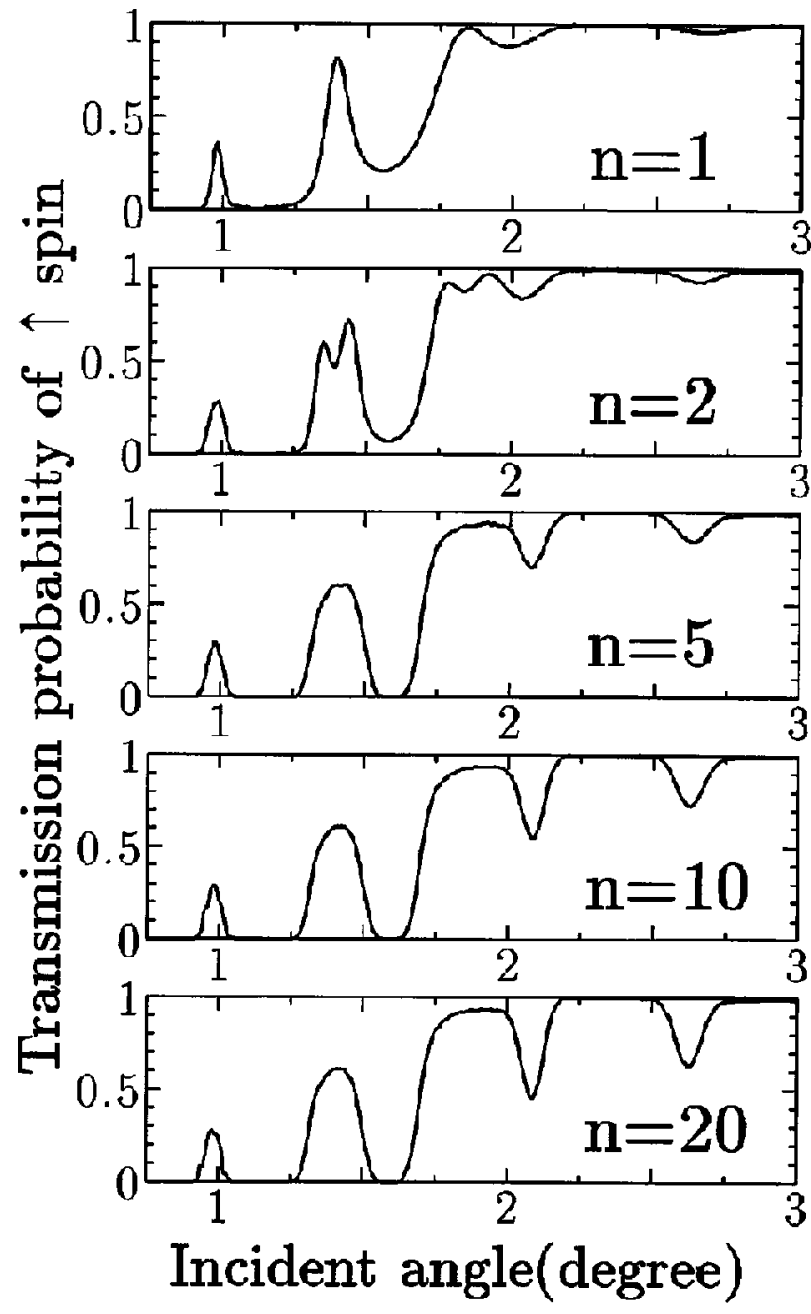

FIG. 10. Simulated transmission probabilities of $\uparrow$ spin neutrons through the $[\mathrm{PA}(20 \mathrm{~nm})-\mathrm{Ge}(40 \mathrm{~nm})]^{n}-\mathrm{PA}(20 \mathrm{~nm})$ Fabry-Perot resonator for $n=1,2,5,10,20$, respectively, as a function of the incident angle.

because the incident wavelength of $1.26 \mathrm{~nm}$ is $0.59^{\circ}$. The measured transmission probabilities are also well reproduced by the theoretical lines derived by solving a one-dimensional stationary Schrödinger equation for each rectangular potential model, including the reduction due to the experimental setup. Since the transmission probability is considered as the average of that for each wavelength in the incident wavelength distribution, we could not find a split of peaks in the multirectangular potential case $(n=10)$. The values of the average nuclear and magnetic potentials evaluated in Fig. 8(b) are also shown in Table I.

Figure 9 shows spin-precession angles of neutrons transmitted through the $[\mathrm{PA}(20 \mathrm{~nm})-\mathrm{Ge}(40 \mathrm{~nm})]^{10}-\mathrm{PA}(20 \mathrm{~nm})$ Fabry-Perot resonator. The measured closed circles are well reproduced by the stationary-state prediction calculated from Eq. (2.4). The broken line indicates the simulated spinprecession angle of neutrons through $[\mathrm{PA}(20 \mathrm{~nm})-\mathrm{Ge}(\infty)]^{10}-\mathrm{PA}(20 \mathrm{~nm})$ as the nonresonance case. The precession angle for $[\mathrm{PA}(20 \mathrm{~nm})-\mathrm{Ge}(\infty)]^{10}-\mathrm{PA}(20 \mathrm{~nm})$ was calculated by adding two kinds of spin-precession angles that are ten times as large as the relative phase differ-

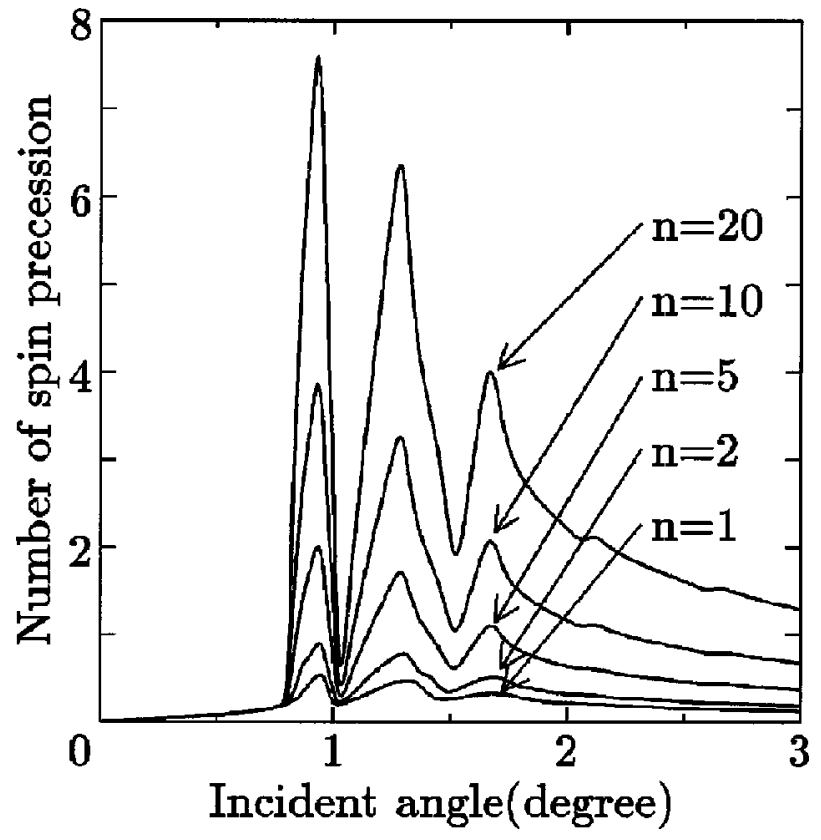

FIG. 11. Simulated spin precession of neutrons through the $[\mathrm{PA}(20 \mathrm{~nm})-\mathrm{Ge}(40 \mathrm{~nm})]^{n}-\mathrm{PA}(20 \mathrm{~nm})$ Fabry-Perot resonators for $n$ $=1,2,5,10,20$, respectively, as a function of the incident angle.

ence due to $\mathrm{PA}(20 \mathrm{~nm})-\mathrm{Ge}(2 \mu \mathrm{m})$ and $\mathrm{Ge}(2 \mu \mathrm{m})-\mathrm{PA}(20$ $\mathrm{nm})-\operatorname{Si}(2 \mu \mathrm{m})$. The incident angle at the peak of the transmission probability corresponds to cross points of the oscillation curve for the gap $40 \mathrm{~nm}$ and $\infty$. From a comparison between Figs. 5(a) and 9, we see that the amplitude of the oscillation curve is almost proportional to the number of the germanium layer (well) although the transmission probability remains constant.

Figure 10 shows the simulated transmission probability of $\uparrow$ neutrons through the $[\mathrm{PA}(20 \mathrm{~nm})-\mathrm{Ge}(40 \mathrm{~nm})]^{n}-\mathrm{PA}(20 \mathrm{~nm})$ Fabry-Perot resonator for $n=1,2,5,10,20$, with the incident wavelength distribution at the NSI-JAERI. The average nuclear and magnetic potentials for Permalloy45 layers are nominal values, 220 and $96.5 \mathrm{neV}$, respectively, and those for the germanium layer were 94.0 and $0 \mathrm{neV}$, respectively. From Fig. 10 it is confirmed that the first and second peaks do not change by increasing the number of wells for $n \geqslant 5$. Figure 11 shows simulated spin-precession angles of neutrons through the $[\mathrm{PA}(20 \mathrm{~nm})-\mathrm{Ge}(40 \mathrm{~nm})]^{n}-\mathrm{PA}(20 \mathrm{~nm})$ Fabry-Perot resonator for $n=1,2,5,10,20$, respectively, with the incident wavelength distribution. It shows that the amplitude of the oscillation curve is proportional to the number of wells whereas the transmission probability remains constant.

Let us consider a question associated with "how long does it take to build up the stationary wave?"' The fact that the spin-precession angle of the transmitted neutron increases with the number of wells provides us with a chance to do a time-dependent experiment for the question related to the shutter problem which is discussed by several authors [22-24]. Considering the spin-precession of neutrons through the $[\mathrm{PA}(20 \mathrm{~nm})-\mathrm{Ge}(40 \mathrm{~nm})]^{100}-\mathrm{Ge}(20 \mathrm{~nm})$ FabryPerot resonator, the traversal time across the resonator for $\downarrow$ spin is expected to be of order $2 \mu \mathrm{sec}$ at the first quasibound state for the $\uparrow$ spin. By oscillating the direction of the applied 
magnetic field for the resonator within $1 \mu \mathrm{sec}$, we can create suddenly vanishing barriers or create opaque barriers for $\uparrow$ and $\downarrow$ spin neutron during neutron stays in the resonator. To measure the spin-precession angle of neutrons through suddenly vanishing barriers or suddenly creates opaque barriers during neutron stays in the resonator, we can estimate that the buildup time of the stationary wave in the resonator takes shorter than the dwell time of neutrons in the resonator. We have the capability of doing the experiment using the techniques of the high-frequency magnetic oscillation field [25] and a very magnetically soft multilayer mirror [26].

\section{CONCLUSIONS}

In this paper the spin-precession angles of neutrons resonantly tunneling through the Fabry-Perot magnetic resonator, which are represented as one-dimensional doublerectangular, triple-rectangular, and multirectangular potential barriers, have been precisely measured as a function of the incident angle. In both resonant tunneling and nontunneling cases, the spin-precession angles were well reproduced by the relative phase difference between the $\uparrow$ and $\downarrow$ spin neutron wave functions derived by solving the one-dimensional Schrödinger equation. The spin-precession angle due to the
Fabry-Perot magnetic resonator shows the oscillation curve as a function of the incident angle. It was experimentally confirmed that the spin-precession angles of the resonant and nonresonant tunneling neutron were the same values at the incident angle for maximum transmission probability of $\uparrow$ spin. This result indicates that the resonant tunneling phenomenon appears when no reflected wave seems to be in the well. It has been experimentally demonstrated that the spinprecession angle due to quantum wells is proportional to the number of wells whereas the transmission probability stays constant.

\section{ACKNOWLEDGMENTS}

One of the authors (M.H.) is grateful to Professor M. Utsuro for his kind support and encouragement. The authors acknowledge practical help and fruitful discussions with $\mathrm{H}$. Tahata. This work was supported by the interuniversity program for the KURRI and JAERI facilities, and financially by the Yamada Science Foundation and a Grant-in-Aid for Scientific Research from the Japanese Ministry of Education, Science, Sports and Culture (Grant Nos. 08874019 and 10440122).
[1] M. Hino, N. Achiwa, S. Tasaki, T. Ebisawa, T. Akiyoshi, and T. Kawai, J. Phys. Soc. Jpn. Suppl. A 65, 201 (1996); 65, 277 (1996).

[2] N. Achiwa, M. Hino, S. Tasaki, T. Ebisawa, T. Akiyoshi, and T. Kawai, J. Phys. Soc. Jpn. Suppl. A 65, 181 (1996).

[3] M. Hino, N. Achiwa, S. Tasaki, T. Ebisawa, T. Kawai, T. Akiyoshi, and D. Yamazaki, Phys. Rev. A 59, 2261 (1999).

[4] K. A. Steinhauser, A. Steyerl, H. Scheckenhofer, and S. S. Malik, Phys. Rev. Lett. 44, 1306 (1980).

[5] A. Steyerl, T. Ebisawa, K. A. Steinhauser, and M. Utsuro, Z. Phys. B 41, 283 (1981).

[6] Y. P. Feng, C. F. Majkrzak, S. K. Sinha, D. G. Wiesler, H. Zhang, and H. W. Deckman, Phys. Rev. B 49, 10814 (1994).

[7] M. Mâaza, B. Pardo, J. P. Chauvineau, A. Raynal, A. Menelle, and F. Bridou, Phys. Lett. A 223, 145 (1996).

[8] M. Mâaza, B. Pardo, J. P. Chauvineau, A. Menelle, A. Raynal, F. Bridou, and J. Corno, Phys. Lett. A 235, 19 (1997).

[9] M. Hino, N. Achiwa, S. Tasaki, T. Ebisawa, T. Kawai, and T. Akiyoshi, Physica B 241-243, 1083 (1998).

[10] V. F. Sears, Neutron Optics (Oxford University Press, New York, 1989).

[11] J. Summhammer, G. Badurek, H. Rauch, U. Kischko, and A. Zeilinger, Phys. Rev. A 27, 2523 (1983).

[12] Neutron Interferometry, edited by U. Bonse and H. Rauch (Clarendon, Oxford, 1979).

[13] S. Yamada, T. Ebisawa, N. Achiwa, T. Akiyoshi, and S. Okamoto, Annu. Rep. Res. React. Inst., Kyoto Univ. 11, 8 (1978).
[14] S. J. Blundell and A. C. Bland, Phys. Rev. B 46, 3391 (1992).

[15] F. Mezei, Z. Phys. 255, 146 (1972).

[16] Neutron Spin Echo, edited by F. Mezei, Lecture Notes in Physics Vol. 128 (Springer, Heidelberg, 1980).

[17] T. Kawai, T. Ebisawa, S. Tasaki, Y. Eguchi, M. Hino, and N. Achiwa, J. Neutron Res. 5, 123 (1997); T. Kawai, T. Ebisawa, S. Tasaki, T. Akiyoshi, M. Hino, N. Achiwa, Y. Otake, and H. Funahashi, J. Phys. Soc. Jpn. Suppl. A 65, 230 (1996).

[18] S. Tasaki, T. Ebisawa, T. Akiyoshi, T. Kawai, and S. Okamoto, Nucl. Instrum. Methods Phys. Res. A 355, 501 (1995).

[19] T. Ebisawa, H. Funahashi, S. Tasaki, Y. Otake, T. Kawai, M. Hino, N. Achiwa, and T. Akiyoshi, J. Neutron Res. 4, 157 (1996); T. Ebisawa, S. Tasaki, T. Kawai, M. Hino, N. Achiwa, Y. Otake, H. Funahashi, D. Yamazaki, and T. Akiyoshi, Phys. Rev. A 57, 4720 (1998).

[20] V. F. Sears, Neutron News 3, 26 (1992).

[21] For example, M. Born and E. Wolf, Principles of Optics, 6th ed. (Pergamon, New York, 1980).

[22] M. Moshinsky, Phys. Rev. 88, 625 (1952).

[23] R. Gähler and R. Golub, Z. Phys. B 56, 5 (1984).

[24] Gastón García-Calderón and Alberto Rubio, Phys. Rev. A 55, 3361 (1997).

[25] T. Kawai, T. Ebisawa, S. Tasaki, M. Hino, D. Yamazaki, H. Tahata, T. Akiyoshi, Y. Matsumoto, N. Achiwa, and Y. Otake, Physica B 241-243, 133 (1998).

[26] M. Hino, T. Kawai, T. Ebisawa, S. Tasaki, and N. Achiwa, Physica B 267-268, 360 (1999). 